OPEN

SUBJECT AREAS:

MICROSCOPY

RESPIRATORY SYSTEM MODELS

RESPIRATION

IMAGING

Received

15 November 2012

Accepted

1 February 2013

Published

18 February 2013

Correspondence and requests for materials should be addressed to

B.M.W. (bmweon@ hotmail.com) or J.H.J. (jhje@postech.ac.kr)

\section{Tracking X-ray microscopy for alveolar dynamics in live intact mice}

\author{
Soeun Chang ${ }^{1,2}$, Namseop Kwon ${ }^{1,3}$, Byung Mook Weon ${ }^{1,2}$, Jinkyung Kim ${ }^{1,3}$, Chin Kook Rhee ${ }^{4}$, \\ Han Sung Choi ${ }^{5}$, Yoshiki Kohmura ${ }^{6}$, Masaki Yamamoto ${ }^{6}$, Tetsuya Ishikawa ${ }^{6} \&$ Jung Ho Je
}

${ }^{1}$ X-ray Imaging Center, Pohang University of Science and Technology, San 31, Hyoja-dong, Pohang, 790-784, Korea, ${ }^{2}$ Department of Materials Science and Engineering, Pohang University of Science and Technology, San 31, Hyoja-dong, Pohang, 790-784, Korea, ${ }^{3}$ School of Interdisciplinary Bioscience and Bioengineering, Pohang University of Science and Technology, San 31 , Hyojadong, Pohang, 790-784, Korea, ${ }^{4}$ Division of Pulmonary and Critical Care Medicine, Department of Internal Medicine, Seoul St. Mary's Hospital, Catholic University of Korea, 505 Banpo-dong, Seocho-Gu, Seoul, 137-701, Korea, ${ }^{5}$ Department of Emergency Medicine, Kyung Hee University Hospital, Seoul, 130-872, Korea, 'RIKEN SPring-8 Center, 1-1-1 Kouto, Sayo-cho, Sayo, Hyogo, 679-5198, Japan.

Here we report a tracking $\mathrm{X}$-ray microscopy $(\operatorname{TrXM})$ as a novel methodology by using upper right lung apices alveoli in live intact mice. By enabling tracking of individual alveolar movements during respiration, TrXM identifies alveolar dynamics: individual alveoli in the upper lung apices show a small size increment as $4.9 \pm 0.4 \%$ (mean \pm s.e.m.) during respiration while their shapes look almost invariant. TrXM analysis in alveolar dynamics would be significant for better understanding of alveolar-based diseases, for instance, ventilator induced lung injury (VILI) in acute respiratory distress syndrome (ARDS).

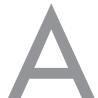

lveoli are elementary gas exchange units in mammalian lungs; their size and shape significantly influence lung function and stability ${ }^{1-3}$. In medical research and practice, mechanical ventilation is of great importance to patients with acute lung injuries, in particular, acute respiratory distress syndrome (ARDS) patients ${ }^{4}$. Various harmful effects followed by ventilation, however, demand the improvement of ventilatory strategies. Despite improvements in clinical outcome, our understanding of potentially protective ventilatory strategies is hampered by the lack of data on alveolar structure ${ }^{2,3}$, specifically alveolar dynamics (the dynamic changes in alveolar sizes and shapes during ventilation $)^{5-7}$. Real-time imaging ${ }^{8}$ of alveoli is essential to identify alveolar dynamics during ventilation ${ }^{2,3}$. Previous studies on real-time alveolar imaging, however, have been limited to alveolar clusters located only at lung lobe surface for thorax-incised mice, using intravital microscopy (IVM) or optical coherence tomography $(\mathrm{OCT})^{5-7}$. Alveolar dynamics could be significantly affected by the intrathoracic pressure change once the thorax is opened ${ }^{5-7}$.

X-ray imaging based on phase contrast and strongly collimated synchrotron X-rays produces images of very high quality, in particular, for living biological specimens ${ }^{9-12}$. Furthermore, synchrotron hard X-rays are highly penetrating and can examine thick specimens with high spatial and temporal resolution, as required here? This approach has been indeed applied to visualize in-vivo the whole lung or small airways of rats and mice during ventilation ${ }^{11,12}$. However real-time imaging of alveolar sacs and ducts in live intact mice during respiration is still largely unexplored. A key problem in real-time X-ray imaging of alveoli is the overall lung movement that also affects individual alveoli movements during respiration significantly, making it very hard to visualize in-vivo alveoli.

The objective of our work is to report a novel and exciting technology, a tracking X-ray microscopy (TrXM) that could visualize alveolar sacs and ducts in two (2-D) and three dimensions (3-D) in live intact mice during respiration. We have presented here proof of concept data by analyzing 100 alveoli in 2-D and 80 alveoli in 3-D in the upper lung apices region. The key idea of this method is to track individual alveoli despite overall lung movement. This approach enabled us to measure the size increment $(4.9 \pm 0.4 \%$ (mean \pm s.e.m.)) of individual alveoli during respiration in the upper lung apices of live intact mice. Each alveolus shows a closed-loop hysteresis between inspiration and expiration. Furthermore we reveal two alveolar shapes: hyper-hemisphere in sacs and hypo-hemisphere in ducts, showing little change in the overall shape of each alveolus during respiration.

\section{Results}

We studied in live intact nude mice the sac alveoli (SA) and duct alveoli (DA) at the apices of the upper right lung lobes (Fig. 1a) that have a minimum lung movement (Fig. 1b) as more or less $100 \mu \mathrm{m}$ during respiration 
a

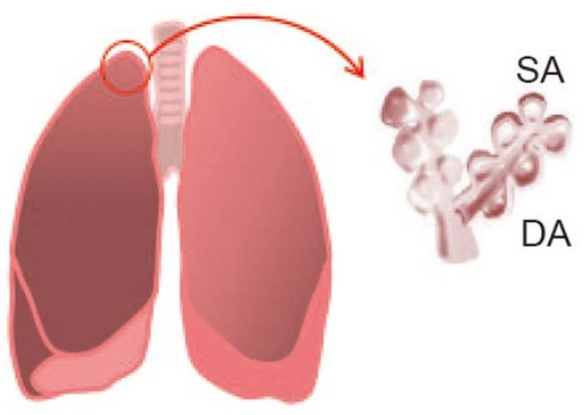

b

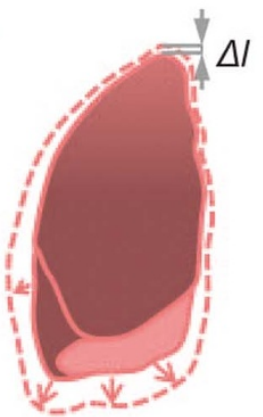

Alveolar imaging

in moving lungs of live mice

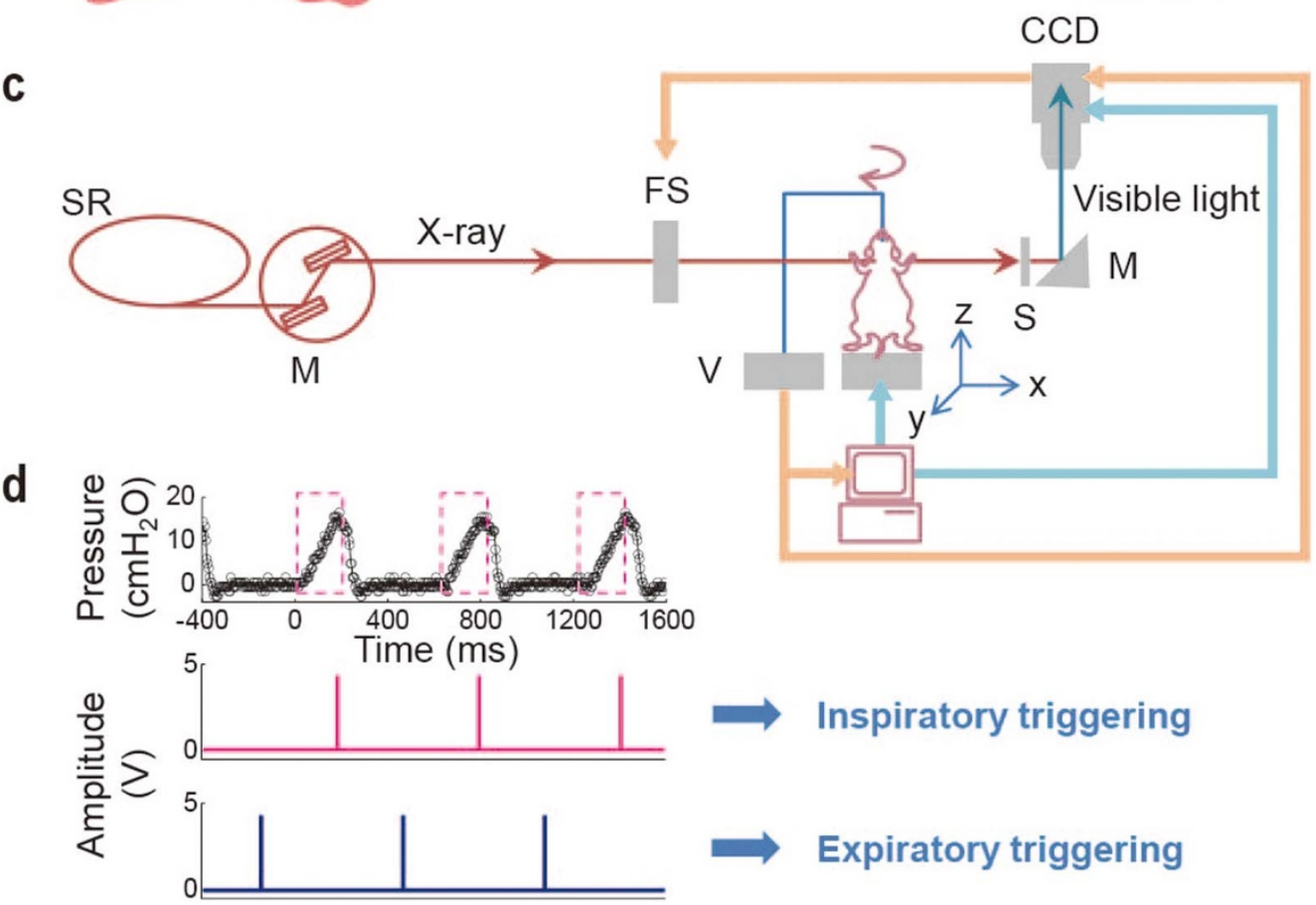

Figure 1 Schematic illustration of TrXM. (a) Schematic of duct alveoli (DA) and sac alveoli (SA). (b) Overall lung movement during respiration. (c) Schematic view of tracking X-ray microscopy (TrXM). The alveolus at the apex top was tracked and maintained at one same coordinate in the field of view during respiration using motorized sample stage. The sample stage, a fast shutter, and a lens-coupled charge-coupled device (CCD) camera were synchronized by a mechanical ventilator. SR: Synchrotron Radiation, M (left): Monochromator, FS: Fast Shutter, V: Ventilator, S: Scintillator, M (right): Mirror. (d) The acquisition sequences of alveolar visualization for microtomography. The pressure at the ventilator is shown at top. Projection images for microtomography were taken at the inspiratory (or expiratory) triggering during $180^{\circ}$ rotation of the sample stage. The normal inspiration/expiration (I/ E) ratio was taken as $1 / 2$.

(Supplementary Fig. 1; Supplementary Video 1). We first describe a TrXM-based tomography that enables us to visualize individual alveoli in 3-D. To resolve the lung movement, the alveolus at the apex top (see the arrow in Supplementary Fig. 1) was tracked with time. The top position was then maintained in one same coordinate of the field of view at both end points of inspiration and expiration, using motorized sample stage. Our microtomography strategy was to take projection image at every end point of inspiration (or expiration) during $180^{\circ}$ rotation of the sample stage (see the inspiratory (or expiratory) triggering in Fig. 1d; Supplementary Videos 2 and 3). The synchronization between the sample stage, a fast X-ray shutter, and a lenscoupled charge-coupled device (CCD) detector was controlled by a mechanical ventilator (Fig. 1c).

Next, we describe a TrXM-based radiography that enables us to visualize individual alveoli in $2-\mathrm{D}$ with high time resolution. We observed that the movement directions of individual alveoli were different each other, which was clearly recognized by tracking individual movement paths during respiration, as clearly seen in Supplementary Fig. 2 (trajectories were recorded for 60 alveoli from 6 mice) and Supplementary Video 4 (from 1 mouse for demonstration). The different trajectory of each alveolus could be presumably due to the interference by adjacent alveoli movements and due to the diversity in duct branch directions. The difference enabled us to recognize the motion of each alveolus during respiration and to track individual alveoli in the overlapped images from numerous alveoli. This tracking permitted real-time size measurements of individual alveoli in 2-D (Fig. 2).

Figure 2a shows representative microradiographs of an alveolus (yellow arrow) in the upper right lung lobe of a mouse during one normal inspiration (0 200 ms)-expiration (200 600 ms) cycle, taken at $8 \mathrm{~ms} /$ frame. Real-time measurement of the alveolar size, defined here as the maximum diameter of an alveolus, was feasible from the microradiographs. The size increment at the end of inspiration (200 ms) was small as 4.7\% in the upper right lung lobe. For statistical analysis, we measured the increments for 100 alveoli with variable sizes over 21 mice, as plotted in Fig. 2b (Supplementary Table 1). The increments show little size dependence, mostly distributed in 0 to $6 \%$ (inset in Fig. 2b). We note that the average size increment in live intact mice is very small as $4.9 \pm 0.4 \%$ (mean \pm s.e.m.) (The line in Fig. $2 b$ indicates the average value.). Recent studies of real-time alveolar imaging in the upper right lung lobe by IVM or OCT for thorax-incised mice showed high area expansion ${ }^{6,7}$ of 
a
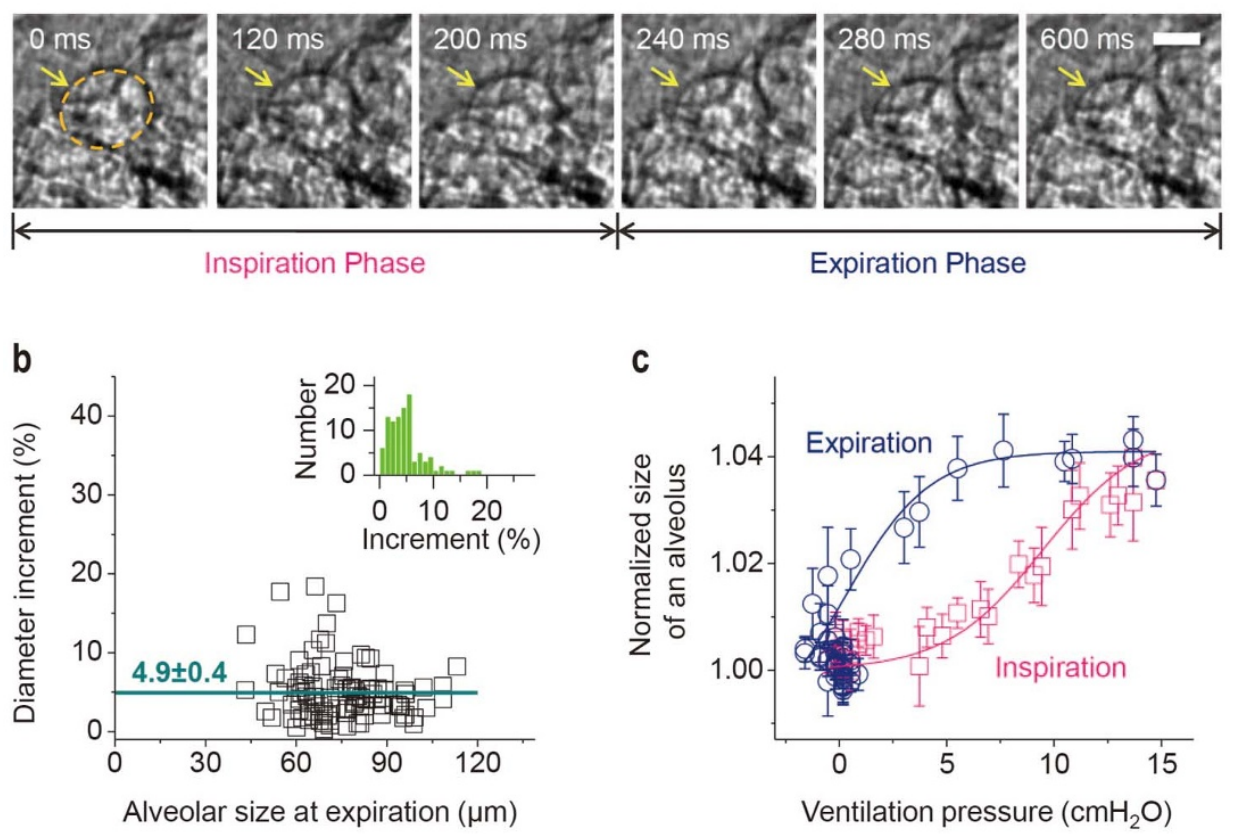

Figure $2 \mid$ Alveolar dynamics in live intact mice. (a) Real-time microradiographs of an alveolus (yellow arrow) in the upper right lung lobe of a mouse during one normal inspiration $(0 \sim 200 \mathrm{msec})$-expiration $(200 \sim 600 \mathrm{msec})$ cycle. Scale bar, $30 \mu \mathrm{m}$. (b) Alveolar size (diameter) increment for 100 alveoli over 21 mice (line: $4.9 \pm 0.4 \%$, mean \pm s.e.m.). Distribution of the alveolar size increment (inset). (c) The size change of an alveolus during 5 inspiration (red solid line)-expiration (blue solid line) cycles. The sizes are normalized to the size measured at the end of expiration.

$\sim 40 \%$ at $24 \mathrm{cmH}_{2} \mathrm{O}$ or very little expansion ${ }^{5}$. In particular, our measurements of the size increment are smaller as $4.9 \%$ than those of $>10 \%$ at $16 \mathrm{cmH}_{2} \mathrm{O}$ by IVM or OCT methods at the right upper lung lobe. This discrepancy is attributed to the thorax opening, which is necessary for IVM or OCT but not adopted in TrXM, because the mechanical environment of alveoli would be changed when the thorax is opened ${ }^{5-7}$.

In Fig. 2c, the pressure-size curve during inspiration and expiration in the tidal volume ventilation was plotted for the alveolus of Fig. 2a. For statistical analysis, the size measurements were performed over 5 respiration cycles (Supplementary Fig. 3). We observe that the alveolus clearly shows a closed-loop hysteresis between inspiration and expiration, similar to whole lung (Supplementary Fig. 4). In the live intact mouse, the compliance of alveoli - defined as fold increase in alveolar volume with a change in ventilation pressure - is low as 0.01 , almost a half of the alveoli compliance of thorax-incised mice ${ }^{5}$ (Supplementary Fig. 4). All the alveoli tested here (taken from 4 mice) showed similar hysteresis behaviors (Supplementary Fig. 5). The low compliance of alveoli in the live intact mice is consistent with the small size increment in individual alveoli.

The alveolar shape can be analyzed by visualizing in 3-D individual alveoli during respiration with the TrXM-based tomography. We successfully reconstructed individual alveoli in a live intact mouse during respiration (Supplementary Fig. 6c and d) and segmented air-filled areas of alveolar ducts and sacs, as demonstrated in the 3-D images of Fig. 3a. As seen in the volume-rendered images (yellow) for one unit of branched ducts, we were able to clearly visualize individual alveoli in sacs and ducts in inspiration (left) and expiration (right) from the upper right lung lobe of the mouse. This enabled us to accurately measure 3-D geometry of each alveolus and to identify alveolar shapes in sacs and ducts in the live intact mouse. We note that there is little change in the overall shape of each alveolus during respiration (see $\mathrm{SA}_{1}$ (sac alveoli), $\mathrm{SA}_{2}, \mathrm{DA}_{1}$ (duct alveoli), and $\mathrm{DA}_{2}$ in inspiration and expiration in Fig. 3a) (Supplementary Videos 5 and 6). a
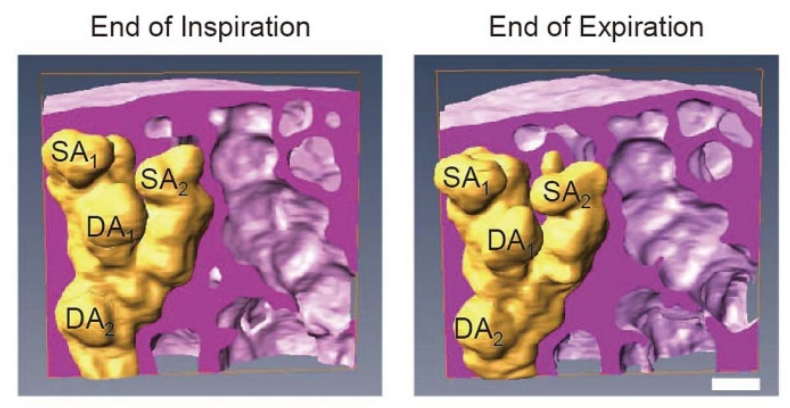

b

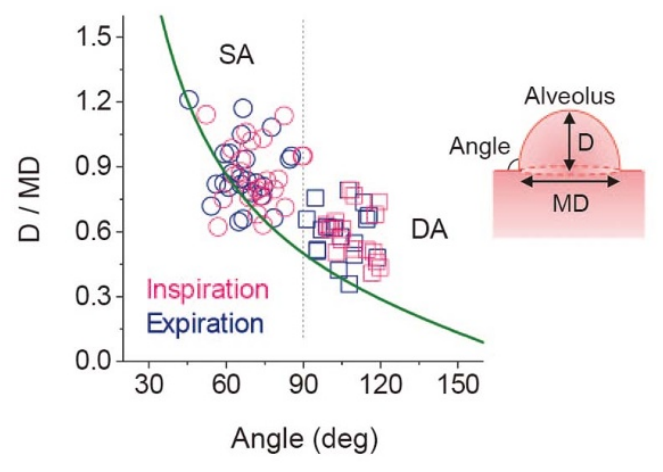

Figure 3 Quantitative analysis of sac and duct alveoli in 3-D geometry. (a) Segmented 3D images of alveoli in ducts and sacs in inspiration (left) and in expiration (right). Scale bar, $50 \mu \mathrm{m}$. (b) The ratio of the alveolar depth (D) and the mouth diameter (MD), D/MD, as a function of alveolar angle, for $\sim 80$ alveoli over 18 ducts with sacs in a live intact mouse. See an alveolus scheme (right). Green line: $\mathrm{D} / \mathrm{MD}$ as a function of alveolar angle assuming spherical cap. D/MD in SA and DA in expiration: $0.87 \pm 0.03$ and $0.59 \pm 0.03$ (mean \pm s.e.m., $P=1.1 \times 10^{-7}$ ); D/MD in SA and DA in inspiration: $0.86 \pm 0.03$ and $0.59 \pm 0.03$ (mean \pm s.e.m., $P=1.3 \times 10^{-7}$ ). 
The ratio of the alveolar depth (D) and the mouth diameter (MD), $\mathrm{D} / \mathrm{MD}$, and the alveolar angle (see the alveolus schematic in Fig. $3 \mathbf{b}$ ), which are important for air flow in breathing ${ }^{13,14}$, were quantitatively measured for $\sim 80$ alveoli over 18 ducts with sacs in Supplementary Fig. 6c and d (Supplementary Videos 7 and 8), as plotted in Fig. 3b. We note that sac alveoli (SA, circles) are clearly distinguished from duct alveoli (DA, squares) by the ratio and the angle. Specifically the average ratio of SA, $0.87 \pm 0.03$ (mean \pm s.e.m.), is higher than that of DA, $0.59 \pm 0.03$ (mean \pm s.e.m.) in expiration (Supplementary Fig. 7; Supplementary Table 2). Meanwhile, D/MDs in SA and DA change little by respiration. Furthermore SA and DA are clearly divided at an alveolar angle, $90^{\circ}$ (dashed line in Fig. 3b). Interestingly the ratio data are lying along a green solid line, which is the ratio value as a function of alveolar angle assuming a spherical cap, within a sphericity of 0.931 \pm 0.022 (mean \pm s.e.m.). This indicates that the overall shape of SA and DA, assessed from the ratio data, is nearly spherical cap. Together with the angle data of SA $\left(\leq 90^{\circ}\right)$ and DA $\left(\geq 90^{\circ}\right)$, this result suggests that the overall alveolar shape in sacs is hyper-hemisphere while that in ducts hypo-hemisphere (Supplementary Fig. 8). Local distortions on the alveolar surfaces in Fig. 3a (Supplementary Fig. 8) are presumably due to adjacent alveoli and diversity in duct branch directions. The overall spherical shape can be also recognized in the projection images of alveoli (Supplementary Fig. 2; Supplementary Video 4). The 3-D results suggest invariance in the alveolar shape during respiration.

\section{Discussion}

Despite experimental improvements in studying alveolar dynamics, previous studies have been limited to alveolar clusters located only at lung lobe surface for thorax-incised mice ${ }^{5-7}$. The strength of the TrXM is its applicability to dynamic studies of thick living organs with high spatial and temporal resolution. In particular, hard X-ray radiography and tomography have the high penetrating power of synchrotron hard X-rays, which enabled us to visualize and measure individual alveoli in live intact mice in upper right lung lobe region during ventilation.

Some technical issues should be resolved in the near future. While we have analyzed 100 alveoli in 21 mice in 2-D and 80 alveoli in one mouse in 3-D, we understand that the measurements are limited to alveoli in the apices of the lungs, where movement is limited with inspiration and expiration. Further improvements of spatial and temporal resolutions in $\operatorname{TrXM}$ would be required to analyze any alveolus in any lung in a live mouse. On the other hand, the mice cannot be positioned in their normal physiological horizontal position. Because of horizontal X-ray beam, the mice were placed upright in a self-developed holder. The upright position, however, allowed precise alignment and rotation of the sample to reduce artifacts in the subsequent tomographic reconstruction ${ }^{12}$. Any possible gravitational effect on the distribution of alveolar size change could be minimized by choosing the apex region of the lung.

How alveoli change size during ventilation in the normal lungs remains uncertain. There are several common hypotheses not restricted to: 1) recruitment and de-recruitment of alveoli; 2) isotropic, balloon-like expansion and contraction of alveoli; 3) alveolar volumetric change accompanied by a shape change; and 4) accordion-like "crumpling" of the alveolar walls $s^{2,3}$. Homogeneous expansion of alveoli with the lung in the upper lung apices would be interesting. We have not observed in respiration any change in alveoli number and any sign of recruitment/de-recruitment ${ }^{2,3}$ and crumpling ${ }^{2,3}$ in the upper lung apices of live intact mice. Our observations might be due to additional volume expansion in alveolar ducts and small airways or to alveolar recruitment/derecruitment in other regions. From the given evidence, we suppose that normal alveoli in the upper lung apices works through a small expansion during respiration and little shape change $e^{5}$. The rest of the lung, especially the bases of the lung where ventilatory changes and movement are greatest, should be further explored to determine the hypotheses about the lung function. We believe that with helps of high penetration and in-vivo imaging, TrXM would be a promising tool to monitor the entire alveoli from whole lungs.

In this study, we successfully achieved an exciting novel technology for in-vivo, non-invasive visualization and quantification of alveoli in live intact mice with TrXM that enabled tracking individual alveolar movements. TrXM-based identification of alveolar dynamics in the upper lung apices showed (i) alveolar size expansion by $4.9 \pm 0.4 \%$ (mean \pm s.e.m.) during respiration and (ii) alveolar shape invariance. TrXM analysis in alveoli would open the way to various studies of alveolar dynamics in live intact animals, with future promise to contribute to better understanding of alveolar-based diseases, for instance, VILI in ARDS.

\section{Methods}

Animal preparation. All experimental protocols were approved by the SPring- 8 Experimental Animals Care and Use Committee. Eight-week-old SPF pathogen-free nude mice (BALB/c-nu, body weight: $20-25$ g, male, SLC Japan Inc., Japan) were anesthetized with $50 \mathrm{mg} \mathrm{kg}^{-1}$ of sodium pentobarbital by intraperitoneal (ip) injection. All the animals studied here had the same birth condition (but they may have slightly different sized lungs). For synchrotron X-ray imaging, tracheotomy was performed with a $22 \mathrm{G} \mathrm{Jelcoß} \mathrm{I.V.} \mathrm{catheter} \mathrm{(Johnson} \mathrm{\&} \mathrm{Johnson} \mathrm{Medical,} \mathrm{Arlington,}$ TX, USA), secured with a suture. The intratracheal catheter was connected to a volume controlled ventilator (Inspira-Advanced Safety Ventilator-Pressure Controlled (ASVP), Harvard Apparatus, USA). The mice were ventilated with room air and were placed in an acrylic tube and fastened in a vertical position. A normal respiration condition for the mice was taken with an inspiration/expiration ratio of $1: 2$, a tidal volume of $160 \mu \mathrm{L} /$ respiration, and a respiratory rate of 100 breaths $/ \mathrm{min}$. After experiments, all the mice were alive.

Real-time X-ray imaging. Real-time X-ray imaging experiments were performed at the RIKEN Coherent X-ray Optics beamline (BL29XU) at SPring-8 (http:// www.spring8.or.jp). SPring-8 is the third-generation synchrotron radiation source in Hyogo, Japan. The X-ray beam produced by an in-vacuum undulator was monochromatized to $15 \mathrm{keV}$ by a double crystal monochromator, and then transported into the experimental hutch, which was located $98 \mathrm{~m}$ from the radiation source. The high coherence of the monochromatic X-rays permitted to observe alveolar boundaries of live intact mice with high visibility by the edge-refraction enhancement $t^{9,11,12}$. The motion blur that can be induced by rapid movements of alveoli during respiration was simply resolved by significantly reducing the exposure time to $8 \mathrm{~ms}$ thanks to the ultrabright synchrotron radiation at SPring-8. Mice were mounted on a high precision motor-controlled stage with the rotational, tilting, and translational resolutions of $0.002^{\circ}, 0.0009^{\circ}$, and $250 \mathrm{~nm}$, respectively. After passing through the sample, the transmitted X-ray beam was converted by a scintillator $\left(\mathrm{CdWO}_{4}\right.$ : Nihon Kessho Koogaku Co. Ltd., Hinata Tatebayashi-City Gunma, Japan) to visible light that was then reflected by a mirror. After magnified by an optical lens, the image was captured by one of the two camera detectors; pco.1600 (The Cooke Corporation, USA; $1600 \times 1200$ pixels) for TrXM-based radiography or Photron Fastcam SA 1.1 (Photron, USA; $1024 \times 1024$ pixels) for TrXM-based tomography.

TrXM-based tomography. The lung movement during respiration is significant as more or less $100 \mu \mathrm{m}$ (Supplementary Fig. 1). To resolve this problem in microtomography, the alveolus at the apex top (see the arrow in Supplementary Fig. 1) was tracked with time. For the tracking of individual alveoli, the top position was maintained at one same coordinate in the field of view using motorized sample stage in the $y$ - and/or z-directions. Then real-time microtomography was performed by controlling the synchronization between the ventilator, the pco.1600 CCD detector, the fast shutter, and the sample stage, as illustrated in Fig. 1c.

The microtomography strategy to track individual alveoli was first to take the projection images at every end point of inspiration during $180^{\circ}$ rotation of the sample stage (see the inspiratory triggering in Fig. 1d), as successfully demonstrated in Supplementary Video 2. Specifically the triggers for the CCD and the X-ray shutters were generated after $190 \mathrm{~ms}$ delay from the onset of each respiration cycle, using a commercial program (Camware). After every image acquisition for $20 \mathrm{~ms}$, the CCD and the X-ray shutters were closed. Then the trigger for the sample rotation by a step angle of $0.36^{\circ}$ were generated after $300 \mathrm{~ms}$ time delay from the onset, using a custom visual basic program developed. Here the onset of each respiration cycle was synchronized by a digital pulse from the ventilator. During the inspiratory triggering, the sample stage stayed on the same position, after translated appropriately to the end point of inspiration. These processes were sequentially repeated for 500 cycles over a circular orbit of $180^{\circ}$. Subsequently the expiratory triggering was carried out at every end point of expiration for the microtomography (Fig. 1d and Supplementary Video 3 ). On this occasion, the time delays for the CCD/X-ray shutters and the sample rotation were 450 and $150 \mathrm{~ms}$, respectively, with the sample stage translated and fixed appropriately to the end point of expiration. 
3-D image acquisition and reconstruction. The projected image set was reconstructed with the standard filter-back projection reconstruction algorithm. Reconstructed slices consisted of $1600 \times 1600$ pixels. The pixel size was $7.4 \times 7.4 \mu \mathrm{m}^{2}$. Vertically stacked 2-D slices were reconstructed for volume-rendered 3-D images using the Amira 5.2 software (Visage Imaging, San Diego, CA, USA). For quantitative analysis in 3-D geometry, reconstructed image stacks of alveoli were manually segmented over alveolar ducts and sacs using the Amira 5.2 software.

Statistical analysis. Data is presented as mean \pm s.e.m. $P$-values were determined by performing a two-tailed $t$-test.

1. Cotes, J. E., Chinn, D. J. \& Miller, M. R. Lung Function: Physiology, Measurement and Application in Medicine 6th edn., (Blackwell Publishing, 2006).

2. Roan, E. \& Waters, C. M. What do we know about mechanical strain in lung alveoli? Am. J. Physiol. Lung Cell Mol. Physiol. 301, L625-L35 (2011).

3. Gatto, L. A., Fluck, R. R. Jr. \& Nieman, G. F. Alveolar mechanics in the acutely injured lung: role of alveolar instability in the pathogenesis of ventilator-induced lung injury. Respir. Care 49, 1045-1055 (2004).

4. The Acute Respiratory Distress Syndrome Network. Ventilation with lower tidal volumes as compared with traditional tidal volumes for acute lung injury and the acute respiratory distress syndrome. N. Engl. J. Med. 342, 1301-1308 (2000).

5. Carney, D., DiRocco, J. \& Nieman, G. Dynamic alveolar mechanics and ventilatorinduced lung injury. Crit. Care Med. 33, S122-S128 (2005).

6. Mertens, M. et al. Alveolar dynamics in acute lung injury: Heterogeneous distension rather than cyclic opening and collapse. Crit. Care Med. 37, 2604-2611 (2009).

7. Meissner, S., Tabuchi, A., Mertens, M., Kuebler, W. M. \& Koch, E. Virtual fourdimensional imaging of lung parenchyma by optical coherence tomography in mice. J. Biomed. Opt. 15, 036016 (2010).

8. Looney, M. R. et al. Stabilized imaging of immune surveillance in the mouse lung. Nat. Methods 8, 91-96 (2011).

9. Hwu, Y. et al. Synchrotron microangiography with no contrast agent. Phys. Med. Biol. 49, 501-508 (2004)

10. Westneat, M. W., Socha, J. J. \& Lee, W. K. Advances in biological structure, function, and physiology using synchrotron X-ray imaging. Annu. Rev. Physiol. 70, 119-136 (2008).
11. Sera, T. et al. Development of high-resolution $4 \mathrm{D}$ in vivo-CT for visualization of cardiac and respiratory deformations of small animals. Phys. Med. Biol. 53, 42854301 (2008).

12. Dubsky, S., Hooper, S. B., Siu, K. K. W. \& Fouras, A. Synchrotron-based dynamic computed tomography of tissue motion for regional lung function measurement. J. R. Soc. Interface 9, 2213-2224 (2012).

13. Harding, E. M. \& Robinson, R. J. Flow in a terminal alveolar sac model with expanding walls using computational fluid dynamics. Inhal. Toxicol. 22, 669-678 (2010).

14. Semmler-Behnke, M. et al. Nanoparticle delivery in infant lungs. Proc. Natl Acad. Sci. USA 109, 5092-5097 (2012).

\section{Acknowledgements}

This research was supported by the Creative Research Initiatives (Functional X-ray Imaging) of MEST/NRF

\section{Author contributions}

S.C., N.K. and J.K. performed experimental work and data analyses. C.K.R. performed medical analysis and interpretation. H.S.C. helped to design the experiments. Y.K., M.Y and T.I. helped to run synchrotron X-ray imaging experiments in SPring-8. B.M.W. supervised data analyses. J.H.J. supervised the work and helped to design the experiments. S.C., B.M.W. and J.H.J. wrote the manuscript. All authors reviewed and edited the manuscript.

\section{Additional information}

Supplementary information accompanies this paper at http://www.nature.com/ scientificreports

Competing financial interests: The authors declare no competing financial interests.

License: This work is licensed under a Creative Commons Attribution-NonCommercial-ShareALike 3.0 Unported License. To view a copy of this license, visit http://creativecommons.org/licenses/by-nc-sa/3.0/

How to cite this article: Chang, S. et al. Tracking X-ray microscopy for alveolar dynamics in live intact mice. Sci. Rep. 3, 1304; DOI:10.1038/srep01304 (2013). 